\title{
Search for a Threatening Target Triggers Limbic Guidance of Spatial Attention
}

\author{
Aprajita Mohanty, Tobias Egner, Jim M. Monti, and M.-Marsel Mesulam \\ Cognitive Neurology and Alzheimer's Disease Center, Feinberg School of Medicine, Northwestern University, Chicago, Illinois 60611
}

The ability to actively locate potential threats in our environment is highly adaptive. To investigate mediating neural mechanisms, we designed a visual search task in which central cues signaled future location and emotional expression (angry or neutral) of a target face. Cues predicting angry targets accelerated subsequent attention shifts, indicating that endogenous signals predicting threatening events can prime the spatial attention network. Functional imaging showed that spatially informative cues activated the fusiform gyrus (FG) as well as frontoparietal components of the spatial attention network, including intraparietal sulcus (IPS) and frontal eye field (FEF), whereas cues predicting angry faces also activated limbic areas, including the amygdala. Anatomically overlapping, additive effects of spatial and emotional cuing were identified in the IPS, FEFs, and FG, regions that also displayed augmented connectivity with the amygdala after cues predicting angry faces. These data highlight a key role for the frontoparietal spatial attention network in the compilation of a salience map that combines the spatial coordinates of an event with its motivational relevance. Furthermore, they suggest that active search for a threatening stimulus elicits amygdala input to the spatial attention network and inferotemporal visual areas, facilitating the rapid detection of upcoming motivationally significant events.

\section{Introduction}

The term spatial attention is used to designate a heterogenous set of neural mechanisms that collectively align the focus of attention with behaviorally relevant events in extrapersonal space. The sensory and motor components of this process, including the mapping of perceptual salience and the implementation of orienting behaviors, have been linked to a distributed frontoparietal network revolving around the frontal eye field (FEF) and the intraparietal sulcus (IPS) (Mesulam, 1981, 1999; Corbetta and Shulman, 2002). Although sensory and motor codes are necessary, they are not sufficient for guiding spatial attention toward behaviorally significant aspects of the environment. The organism often encounters events that are highly significant not because of their sensorial intensity but because of learned stimulus-outcome associations or their intrinsic biological significance. Hence, the spatial attention system also needs to consider endogenous information about the motivational value of external stimuli.

In humans, evolutionary contingencies have conferred a special status to the processing of threatening and fearful facial features. For example, angry faces have been shown to be particularly effective in capturing attention exogenously to their location (Mogg and Bradley, 1999; Eastwood et al., 2001; Armony and Dolan, 2002; Tipples et al., 2002; Pourtois et al., 2004). In

\footnotetext{
Received March 6, 2009; revised June 10, 2009; accepted June 29, 2009.

This work was supported by National Institutes of Health Grant NS30863 (M.-M.M.) from the National Institute of Neurological Disease and Stroke (NINDS). A.M. was supported by NINDS Training Grant T32 NS047987.

Correspondence should be addressed to Dr. Aprajita Mohanty, Cognitive Neurology and Alzheimer's Disease Center, Feinberg School of Medicine, 320 East Superior Street, Searle 11-579, Chicago, IL 60611. E-mail: a-mohanty@northwestern.edu.

DOI:10.1523/JNEUROSCI.1170-09.2009

Copyright $\odot 2009$ Society for Neuroscience $\quad$ 0270-6474/09/2910563-10\$15.00/0
}

addition, motivationally salient information can also be used endogenously, for example, when searching for an angry face in a crowd (Hahn and Gronlund, 2007). Such anticipatory search behaviors, aiming to detect sources of potential threat, are part of naturalistic behaviors in a wide range of habitats from the jungle to social gatherings. However, the way in which previous motivational information is integrated with the sensory-motor components of spatial attention to guide visual search is poorly understood.

One way in which endogenous motivational information could guide spatial attention is via input to the spatial attention network from limbic regions involved in coding motivational significance, such as the amygdala. Frontoparietal regions receive monosynaptic projections from limbic parts of cingulate gyrus, which in turn is connected to the amygdala, raising the possibility that the cingulate gyrus serves as a conduit for information on motivational salience used by the spatial attention network (Mesulam et al., 1977; Mohanty et al., 2005; Small et al., 2005). The spatial attention network may then integrate the spatial and motivational sources of information to form a search template ("top-down salience map") for biasing visual neurons in preparation for the search process (Egner et al., 2008). However, functional integration of spatial and motivational a priori search information of this kind has not previously been demonstrated.

Therefore, in the present study, we examined how the brain uses foreknowledge regarding intrinsically significant stimuli to guide spatial attention by using a visual search task in which predictive cues offered probabilistic information related to the location and emotional salience of an upcoming target. In contrast to previous experiments that have investigated responses to the physical presence of motivationally relevant targets (i.e., 
exogenous attention), the present experiment was designed to explore the response of the spatial attention network to the anticipation of a threatening stimulus (i.e., endogenous attention).

\section{Materials and Methods}

\section{Participants}

Fifteen right-handed volunteers (seven women; mean/SD age, 27/3.80 years) participated in the study. Participants had normal or correctedto-normal vision and were screened for a history of psychiatric and neurologic illness or contraindications for functional magnetic resonance imaging (fMRI). All participants gave written informed consent before participation, and the study procedures were approved by the Northwestern University Institutional Review Board. Two participants, one outlier in behavioral performance and one showing fMRI-related artifacts, were excluded, resulting in a final $N$ of 13 .

\section{Stimuli and design}

Participants performed a cued visual search task that independently varied the degree of spatial and emotion-related information available regarding the upcoming search target. The task programming, stimulus delivery, and recording of behavioral responses were performed with Presentation software (Neurobehavioral Systems). While performing the task, participants were asked to fixate on a central diamond (subtending a $1.6^{\circ}$ visual angle) that remained on the screen for the complete duration of the task. Each trial consisted of a cue period and a visual search (target) period (see Fig. $1 \mathrm{~A}$ ). The cues (subtending a $1.6^{\circ}$ visual angle) presented in the cue period were designed to systematically bias the search for the target in the search array that was presented during the visual search period. The participants task was locate the "target," a tilted face among upright faces presented in the search array and indicate direction of tilt. Detection of this target was aided by valid cues providing two types of information with $90 \%$ accuracy: spatial information that was conveyed by a highlighting of either the left or right side (spatially informative) or the entire (spatially uninformative) central fixation diamond, and emotional information that was conveyed by a change in color to red (emotionally negative), black (emotionally uninformative), or green (emotionally neutral) of the highlighted portion of the cue (see Fig. $1 B$ ). This resulted in a two spatial cue information (directional and nondirectional) by three emotional cue information (negative, uninformative, and neutral) factorial design (see Fig. 1B). For example, a cue could inform a participant that the target face has a $90 \%$ probability of appearing on the right side of space and $90 \%$ probability of being an angry expression (see Fig. 1A). This cue remained on the screen until the appearance of the target array to avoid engaging working memory. In addition to emotionally and spatially informative cues (targets appearing on the side and of the emotion indicated by the cue) and uninformative cues (no information regarding the target identity), which constituted $80 \%$ of trials, the experimental design also included emotionally and spatially invalid cues (targets appearing on the side opposite and/or of the emotion opposite to that indicated by the cue), which constituted $20 \%$ of the total trials.

The target search array consisted of a central fixation diamond flanked by four peripherally placed facial stimuli. The face stimuli were selected from the NimStim Set of Facial Expressions, which is a standardized database of photographs of professional actors portraying various emotions (http://macbrain.org/resources.htm). All images involved a single actor depicting neutral and angry facial expressions and were matched for luminance and contrast and subtended a horizontal visual angle of $2.7^{\circ}$ and a vertical angle of $3.6^{\circ}$. In addition, the face stimuli were converted to grayscale, cropped to an oval shape to remove hair, neck, and background information. Two faces (one angry and one neutral) were placed to the left and two faces (one angry and one neutral) were placed to the right of the central fixation at an eccentricity of $5.5^{\circ}$ on a gray background (red, blue, green: 149, 149, 149). On each trial, one of the face stimuli (the "target") was tilted by $5^{\circ}$ to either the left or right (see Fig. $1 \mathrm{~A}$ ), and the participant's task was to locate the tilted face and indicate direction of tilt by pressing a button with either the right index or middle finger. Thus, for any given target array, the participant could use a combination of the spatial and emotion-related information provided by the preceding cue to identify the target stimulus. The highlighting and color of each cue, the configuration of the facial stimuli, the identity of the target face, and the location of the target face were all varied randomly from trial to trial. Finally, it is important to note that, although the information provided by the cue facilitated the search for the target face, the information was not predictive of the correct response (i.e., left or right tilt) associated with the target and thus did not prime the correct response.

The timing parameters in the study were optimized to enable us to statistically separate blood oxygenation level-dependent (BOLD) responses related to cue processing from those related to target processing. To dissociate cue-related activity from subsequent target-related activity, the cue interval duration was jittered, ranging from 3 to $7 \mathrm{~s}$ (in $1 \mathrm{~s} \mathrm{bins),}$ along a pseudoexponential distribution of $3 \mathrm{~s}$ (50\%), $4 \mathrm{~s}(25 \%), 5 \mathrm{~s}$ (12\%), 6 s (6\%), and 7 s (6\%) intervals (Ollinger et al., 2001a,b; Wager and Nichols, 2003). To further decorrelate cue from target processing, a proportion of the trials (20\%) consisted of "catch trials," in which a cue stimulus was not followed by a target (Ollinger et al., 2001b). During data analysis, variance accounted for by the cue versus target period was modeled by separate regressors to examine their respective contributions. The target stimuli lasted until the subject's response. Finally, to dissociate target-related activity from subsequent cue-related activity, the intertrial interval duration was varied in the same way as the cue interval duration. Evidence from both computational modeling and empirical results suggest that the temporal jittering proposed for the current study ensures a reliable distinction of BOLD signal attributable to successive events (Dale, 1999; Corbetta et al., 2000; Ollinger et al., 2001a,b; Wager and Nichols, 2003).

In the scanner, the participants viewed the task via a mirror attached to the head coil. Using a liquid crystal display projector attached to the stimulus presentation computer, stimuli were backprojected onto a translucent screen that the participants viewed through a mirror. Participants performed a total of eight runs, consisting of 46 trials each, split into two experimental sessions. The total task lasted for $50 \mathrm{~min}$ including interspersed rest periods. Participants were given two nonmagnetic button boxes, which enabled recording of their reaction time (RT) data.

\section{Eye movement data acquisition}

To control for eye movement-related artifacts, participants were asked to maintain fixation throughout the cue period. Eye-tracking data were acquired to confirm that cue-related BOLD responses reflected topdown biasing of covert attention attributable to spatial and emotional cue-related information and were not contaminated by eye movements. Eye position was monitored during scanning with a magnetic resonancecompatible infrared eye tracker (ASL model 540; Applied Science Group), which monitors and records the relative positions of the pupil and corneal reflection, in reference to the visual display.

\section{fMRI data acquisition}

Functional images were acquired with a 3 tesla Thermo Fisher Scientific Trio whole-body magnetic resonance imaging system using a 12-channel birdcage head coil. In each of the eight runs, 209 images were acquired using echoplanar T2-weighted sequence [repetition time (TR), $2.2 \mathrm{~s}$; echo time (TE), $20 \mathrm{~ms}$; flip angle, $80^{\circ}$; field of view (FOV), $220 \times 220$ $\mathrm{mm}$; matrix, $128 \times 128$ voxels]. Each image consisted of 35 contiguous axial slices (slice thickness, $3 \mathrm{~mm}$; in-plane resolution, $1.7 \times 1.7 \mathrm{~mm}$ ) acquired parallel to the anterior and posterior commissures. Six dummy images were collected at the beginning of each functional run to allow the BOLD response to reach a steady state and were excluded from additional processing and analysis. After the fMRI acquisition, a 176 slice T1weighted MPRAGE (magnetization-prepared rapid acquisition gradient echo) structural sequence (TR, $19 \mathrm{~ms}$; TE, $5 \mathrm{~ms}$; flip angle, $20^{\circ}$; FOV, $220 \times 220 \mathrm{~mm}$ ) of a slice thickness of $1.5 \mathrm{~mm}$ and in-plane resolution of $0.86 \times 0.86 \mathrm{~mm}$ was acquired and used to register the participant's functional data into standard space.

\section{Data analyses}

Behavioral analyses. Behavioral data were screened for statistical outliers using the boxplot technique and trials with outlier RT performance were excluded from additional analyses. Mean RTs computed for each spatial 
and emotional cue type were subjected to a repeated-measures ANOVA with target valence, emotional cue information, and spatial cue information as factors. Significant interactions were examined further with simple effects tests. Accuracy data were also examined and only subjects who met the $80 \%$ accuracy threshold were included in the study, which led to the exclusion of one subject.

Eye movement analyses. Eye data were analyzed with ASL Eyenal software. Artifacts related to blinking were filtered out. We were able to acquire valid eye movement data for all the runs on three subjects and for $20 \%$ of the runs on eight other subjects. For all the subjects, eye movements were monitored on-line by the experimenters and none of the subjects reported having trouble in remaining fixated on the cue. For analyses of eye movement data, an area of interest of $3^{\circ}$ diameter from the center of the fixation cue (which subtended a $1.6^{\circ}$ visual angle) was defined as the fixation zone. For each subject, the amount of time spent in this fixation zone between cue and target presentation was calculated. Results showed that subjects were able to maintain fixation on the cue for $91.71 \%$ ( \pm 8.77$)$ of the time. Furthermore, for a sample of 420 artifactfree cue periods, we were able to show that success at fixating did not vary with cue type (range, 95.05-96.7\%).

fMRI analyses. fMRI data were analyzed using the SPM5 software (Wellcome Department of Cognitive Neurology, London, UK) running under the MATLAB environment (Mathworks). Functional images for each participant were corrected for slice timing, spatially realigned for correction of motion artifacts, coregistered to that participant's mean functional image and high-resolution anatomical T1 image, spatially normalized using Montreal Neurological Institute (MNI) template brain, and smoothed with an isotropic Gaussian kernel of full width half-maximum of $6 \mathrm{~mm}^{3}$. The first five volumes of each run were discarded before estimation of the statistical model. Runs with more than one voxel of scan-to-scan movement were excluded from additional analysis resulting in exclusion of one run from one subject.

For each subject, the canonical hemodynamic response function (HRF) approximating the temporal course of the BOLD HRF to emotionally negative, uninformative, and neutral cues were modeled separately for the entire duration of the spatially informative and uninformative cues. The model also included regressors that coded for target-related activity separately for negative and neutral targets and a regressor that coded for incorrect trials. Finally, the model included a $128 \mathrm{~s}$ high-pass filter to remove low-frequency fluctuations and an $\mathrm{AR}(1)$ model to account for temporal nonsphericity attributable to autocorrelations. This model yielded a per-voxel parameter estimate $(\beta)$ map representing the magnitude of activation associated with each trial type. The cue-related parameter estimates were entered into a second-level random-effects analysis using a SPM5 implemented $2 \times 3$ mixed ANOVA with spatial cue information (informative vs uninformative) and emotional cue information (negative, uninformative, and neutral) as fixed factors and subjects as a random factor. This analysis yielded regions that were significantly modulated by spatial cue-related effects, emotional cue-related effects, as well as an interaction of the two. A spatial cluster extent threshold was used to correct for multiple comparisons using AlphaSim (Alpha Simulations) with 1000 Monte Carlo simulations and taking into account the entire echoplanar imaging gray-matter matrix (http:// afni.nimh.nih.gov/pub/dist/doc/manual/AlphaSim.pdf). This procedure yielded a combined threshold of $p<0.005$ and cluster size of 50 contiguous voxels, which is equivalent to a corrected threshold of $p<$ 0.05 . For clusters of activation identified via this threshold, parameter estimates averaged across the whole cluster were extracted using the Marsbar toolbox (http://marsbar.sourceforge.net/) for each condition. Bar graphs and additional statistics reported in Results for these averaged parameter estimates are for descriptive purposes.

Since we had specific a priori hypotheses regarding the role of the amygdala in the encoding of emotional valence of cues and the posterior cingulate cortex (PCC) in mediating the interaction of spatial and emotional cuing and because these regions are anatomically relatively small, the extent threshold was relaxed to a cluster size of 10 contiguous voxels to examine amygdala and PCC activation for main effects of emotional cuing and interaction of emotional and spatial cuing, respectively. In addition to examining main and interaction effects, a standard con- junction analysis (Friston et al., 2005; Nichols et al., 2005), as implemented in SPM5, was performed to reveal the regions activated in common between the spatial and emotional cue-related effects.

Next, we conducted regression analysis to examine brain areas whose activity correlated with behavioral measures of attentional shifting. First, the behavioral measure of attentional shifting or cue benefit score (the degree to which a valid directional cue benefits performance) was calculated using Equation 1 as follows:

$$
\frac{\overline{\mathrm{RTN}}-\mathrm{RTV}_{i}}{\sigma_{\mathrm{RTN}}} .
$$

RTN is the mean reaction time for the nondirectional trials, $\mathrm{RTV}_{i}$ is the reaction time for each validly cued trial, and $\sigma_{\mathrm{RTN}}$ is the SD of the nondirectional trials. The cue benefit scores were calculated separately for each emotional cue type (negative, uninformative, and neutral). Next, the cue benefit scores were used to predict variations in the HRF during the validly cued trials. For this purpose, another statistical model was estimated in SPM5 to examine brain areas whose activity was associated significantly with cue benefit scores. This model was identical with the one outlined above except for the addition of a condition-specific regressor that modeled the benefit scores derived from the cues as a continuous factor. This regressor allowed us to identify voxels that specifically show a significant correlation with cue benefit scores on validly cued trials, separately for emotionally negative, uninformative, and neutral cues, resulting in three $\beta$ maps. Next, we examined how the correlation between neural activity and the speed of attentional shifts varied for negative, uninformative, and neutral cues by comparing corresponding $\beta$ maps. These maps were forwarded to a second-level random-effects analysis using a SPM5 implemented one-way mixed ANOVA with emotional cue information (negative, uninformative, and neutral) as fixed factors and subjects as a random factor. Since our aim was to investigate whether the spatial attention network mediates anticipatory shifts in spatial attention differently for emotional and nonemotional cues, we focused our analyses on regions of the spatial attention network.

Finally, we examined the task-dependent changes in connectivity between regions involved in emotional and spatial biasing of attention using psychophysiological interaction (PPI) analyses (Friston et al., 1997). The amygdala and PCC, which have been shown to be involved in motivation-related modulation of attention, served as seed regions for the PPI. The deconvolved time-series data for amygdala and PCC were extracted from each participant's normalized data, based on a sphere of radius $6 \mathrm{~mm}$ around the peak activation voxel from the group analyses for main effect of emotion. The product of this activation time-series data and the psychological vector of interest (negative emotional cues uninformative emotional cues) resulted in the psychophysiological interaction term, which was then convolved with the HRF (Gitelman et al., 2003). New SPMs with the physiological variable (e.g., amygdala activity), psychological variable, and their interaction as regressors were computed for each subject. These subject level PPI SPMs were then entered into a random-effects group analyses using $t$ test. Since we were specifically interested in how limbic inputs into the spatial attention network and visual areas facilitates search for motivationally salient targets, we examined functional coupling in regions of the spatial attention network showing a conjunction of emotional and spatial biasing using a threshold of $p<0.05$, uncorrected.

\section{Results}

In the present study, we examined the effects of spatial and emotion-related top-down biasing of attention by asking participants to perform an event-related fMRI task in which centrally presented cues biased the search for an attentional target. The target was a slightly tilted face among upright faces presented in a visual search array and the participant's task was to indicate the direction of the tilt (Fig. 1A). The detection of this target was aided by central cues providing information regarding its likely spatial location and emotional expression. The degree of spatial and emotion-related information provided by a cue regarding 
the upcoming search target was independently varied in a $2 \times 3$ factorial design with spatial cue information (informative and uninformative) and emotional cue information (negative, uninformative, and neutral) as factors (Fig. $1 B$ ). Emotionally and spatially informative cues could either be $90 \%$ valid (targets appeared on the side and of the emotional valence indicated by the cue) or invalid (targets appeared on the side opposite and/or of the emotional valence opposite to that indicated by the cue). Uninformative cues provided no information regarding the target identity. These different trials were presented in randomized event-related manner while fMRI data were recorded.

The main aim of the study was to examine how the brain represents spatial and emotion-related foreknowledge as well as an integration of the two. This was done by exploring the main, interaction, and conjunction effects of spatial and emotional cue-related information. Next, we examined whether regions of the spatial attention network mediate anticipatory spatial attention differentially for emotional and nonemotional cues. This was done by examining the correlation between neural activity and speed of attentional shifts induced by emotional and nonemotional cues. We also examined the role of limbic input to the spatial attention network and inferotemporal visual areas in facilitating the rapid detection of upcoming motivationally significant events. This was done by examining task-dependent changes in connectivity between limbic and spatial attention network regions using PPI analyses.

\section{Behavioral}

We first investigated whether cue-related knowledge regarding the spatial location and the emotional nature of the upcoming target facilitated behavioral performance. We expected response times (RTs) to vary as a function of cue information and cue validity. Specifically, in contrast to spatially uninformative cues, spatially informative cues would be associated with faster RT on validly cued trials (spatial cue benefits) and with slower RT on invalidly cued trials (spatial cue cost). Similarly, it was expected that, compared with emotionally uninformative cues, emotionally negative cues would be associated with faster RT on validly cued trials (emotional cue benefits) and with slower RT on invalidly cued trials (emotional cue cost). Since negative, but not neutral emotional information is motivationally salient and hence effective in providing top-down biasing of attention (Hahn and Gronlund, 2007; Lamy et al., 2008), the same effect was not expected for neutral emotional cues.

As depicted in Figure 1, $C$ and $D$, and Table 1, we observed the hypothesized pattern of results. These effects were formally tested with a three-way repeated-measures ANOVA with target valence (negative vs neutral), spatial cue information (directionally insignificant difference $(p<0.05)$.

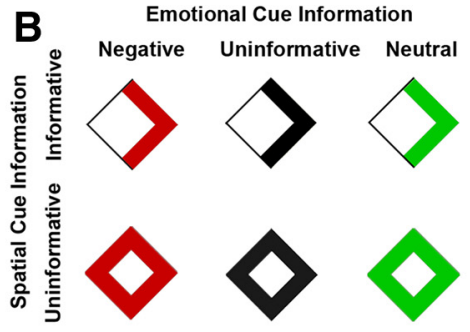

D

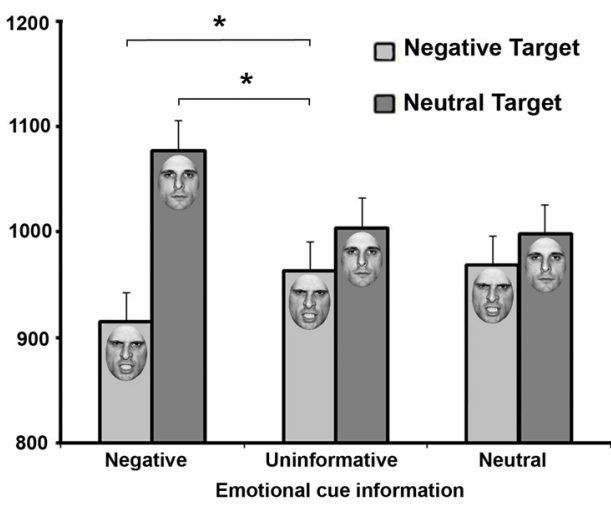

Figure 1. Experimental task and behavioral results. $\boldsymbol{A}$, Participants saw a cue followed by a target search array consisting of a central fixation diamond flanked by four peripherally placed facial stimuli. Two faces (one angry and one neutral) were placed to (he placed to the right of the central fixation. Participants were instructed to

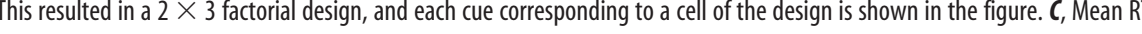
within-subject SEM (wsSEM). The asterisk indicates statistically significant difference $(p<0.05)$. D, Mean RT displayed as a function of attentional target and emotional cue information (collapsed across informative and uninformative levels of spatial cue

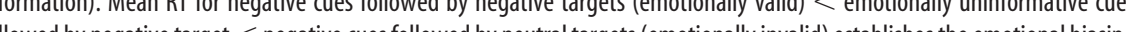
of attention. Similar effects were not noted for neutral cues. Error bars represent wsSEM. The asterisk indicates statistically

formative vs uninformative), and emotional cue information (negative, uninformative, and neutral) as factors. Results showed a significant main effect of spatial cue information with faster detection of targets signaled by directionally informative than uninformative cues (Fig. $1 C)\left(F_{(1,12)}=19.49\right.$; $p<0.001$ ), indicating effective spatial biasing of attention in the present study. A separate one-way repeated-measures ANOVA confirmed results depicted in Figure $1 C$ with spatially valid $\mathrm{RT}<$ spatially uninformative RT $<$ spatially invalid RT $\left(F_{(1,13)}=16.75 ; p<0.001\right.$; liner trend, $\left.F_{(1,13)}=19.83, p<0.001\right)$. In the overall three-way ANOVA, we also found a significant main effect of target with faster detection of negative compared with neutral targets $\left(F_{(1,12)}=27.16\right.$; $p<0.001$ ), reflecting bottom-up capture of attention by negative targets. There was no significant main effect of emotional cue information $\left(F_{(2,11)}=0.505 ; p>0.1\right)$; however, there was a significant target valence by emotional cue information interaction $\left(F_{(2,11)}=4.11 ; p<0.05\right)$. Additional examination of this interaction using the simple effects tests showed that, compared with emotionally uninformative cues, valid negative cues (negative cue followed by a negative target) were associated with faster RT (indicating cue benefits), whereas invalid negative cues (negative cue followed by a neutral target) were associated with slower RTs 
Table 1. Reaction time

\begin{tabular}{|c|c|c|c|c|c|c|}
\hline \multirow[b]{2}{*}{ Spatial cue information } & \multicolumn{3}{|c|}{ Negative-target emotional cue information } & \multicolumn{3}{|c|}{ Neutral-target emotional cue information } \\
\hline & Negative (ms) & Uninformative (ms) & Neutral (ms) & Negative (ms) & Uninformative (ms) & Neutral (ms) \\
\hline Informative & $881.42(144.38)$ & $939.17(143.57)$ & $1001.48(310.03)$ & $1155.57(358.92)$ & $999.89(275.5)$ & $968.21(195.78)$ \\
\hline Uninformative & 1003.55 (199.81) & $1050.75(214.07)$ & $1073.96(304.83)$ & $1181.16(399.34)$ & $1151.95(339.85)$ & $1113.35(245.44)$ \\
\hline
\end{tabular}

Data are expressed as mean (SD).
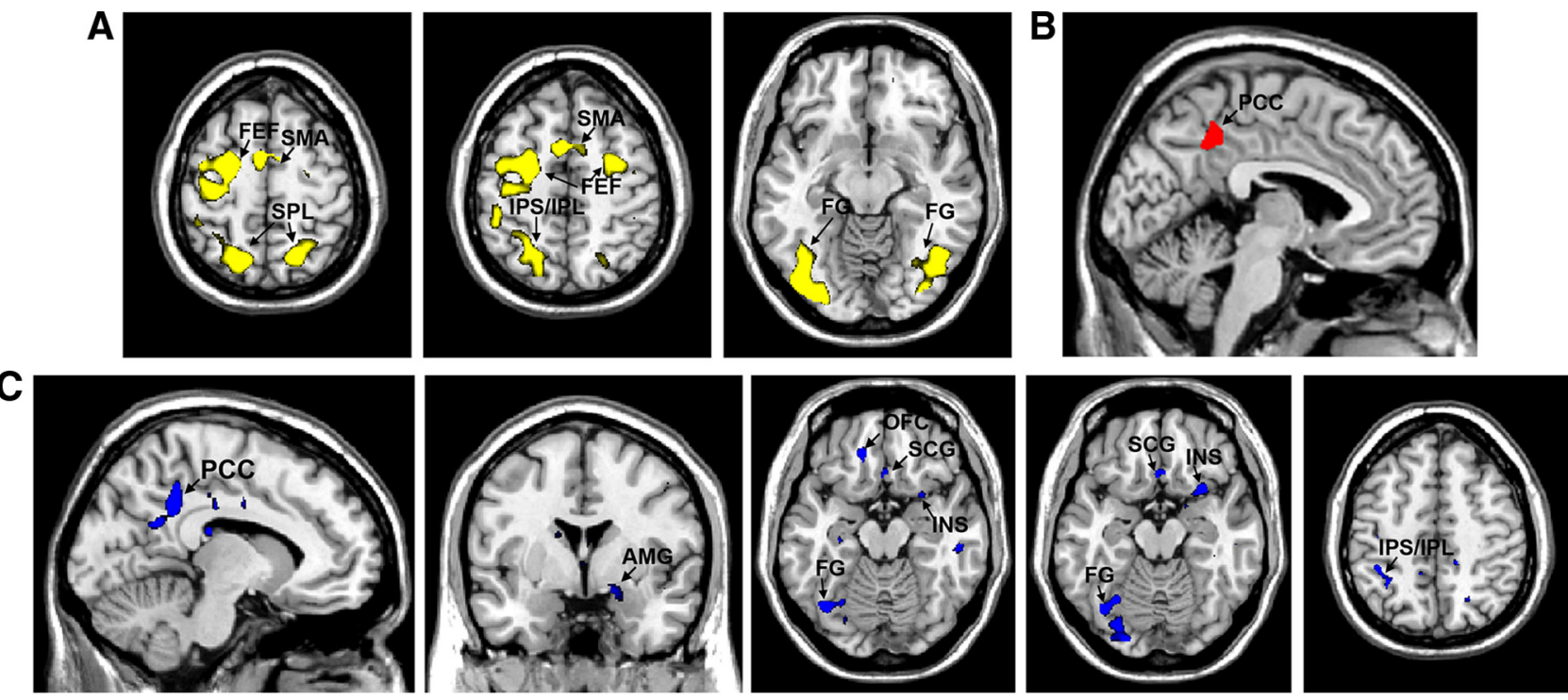

Figure 2. Brain areas that are responsive to spatial and emotional cue information. $\boldsymbol{A}$, Images from the group random-effects analyses depict regions that showed greater activation for spatially informative compared with spatially uninformative cues (collapsed across levels of emotional cue information). Axial sections show activity in FEF, supplementary motor areas (SMA), SPL, IPS/IPL, and FG. $\boldsymbol{B}$, Interaction of spatial and emotional cue-related information in the PCC. PCC showed increased activity for spatially informative cues only when the attentional target was high in emotional or motivational relevance. C, Images depict regions showing negative $>$ uninformative $>$ neutral cue-related activity (collapsed across levels of spatial cue information). Sagittal and axial sections display activity in PCC, amygdala (AMG), orbitofrontal cortex (OFC), and subcallosal gyrus (SCG). Additional brain areas include insula (INS) and FG.

(indicating cue costs; all values of $p<0.05$ ) (Fig. $1 D$ ). Importantly, this effect did not generalize to emotionally neutral cues, indicating that it reflects a relatively specific effect of salient negative cue-related information on the biasing of attention.

To confirm that greater cue benefits derived from valid negative cues (negative cue followed by a negative target) compared with uninformative emotional cues (uninformative cue followed by negative or neutral target) were not attributable solely to target-related differences between the two trial types, we compared the benefits from valid negative cues to benefits from uninformative emotional cues followed by negative targets only. Results showed that, even after controlling for target-related effects, the valid negative cues benefited performance relative to the emotionally uninformative cues $\left(t_{(13)}=2.51 ; p<0.05\right)$ (Fig. $1 D)$. Similarly, controlling for target-related effects, we found that invalid negative cues (negative cue followed by neutral target) impeded performance relative to uninformative cues followed by neutral targets $\left(t_{(13)}=2.60 ; p<0.05\right)$. Finally, we examined the interaction of spatial and emotional cue information to determine whether the effects of emotional cuing differed for levels of spatial cuing, or vice versa. Results showed that the spatial by emotional cue information interaction was not significant $\left(F_{(2,11)}=1.67 ; p>0.1\right)$.

We also conducted analysis to compare the magnitude of cuing costs associated with spatial and negative emotional cues. For this purpose, we did a traditional "cue validity effect" analysis in which valid trial RTs were subtracted from invalid trial RTs. The resulting difference scores for spatial and emotional cue information were then compared with a paired $t$ test. Results showed that the type of information (spatial or emotional) did not differentially impact the cue validity effects $\left(t_{(12)}=0.89 ; p>0.1\right)$ indicating comparable benefits from these two sources of information. In summary, our behavioral results demonstrate that both spatial and emotional cues aided visual search and were effective in inducing comparable and additive benefits on visual search performance. Most importantly, cues predicting angry faces led to faster attentional shifts than uninformative cues followed by angry faces, indicating that the emotional cue-induced acceleration in performance was endogenously mediated and not dependent on bottom-up stimulus features.

\section{fMRI}

Spatial and emotional cue-related effects

An important goal of the present study was to examine how emotional and spatial cue-related information is represented and integrated in the human brain. Hence, we focused our fMRI analyses on the preparatory cue interval. BOLD responses in this interval reflect anticipation of attentional targets that are not contaminated with bottom- up stimulus factors, target detection, response selection, or visual search-related processes (Kastner et al., 1999; Corbetta et al., 2000; Hopfinger et al., 2000). We ensured adequate separation of cue and target-related effects by jittering the time interval between the presentation of cue and target trials and by using catch trials (described in Materials and Methods). In addition, spatial and emotional cue information was modeled as separate from target-related factors, thus, statistically controlling for any target-related variance. We assessed the success of these strategies by calculating correlations between cue 
and target regressors, as a function of emotional and spatial cuing conditions, which yielded low, nonsignificant correlation values (ranging from -0.01 to 0.25 ). Finally, we controlled for oculomotor activity in the cue interval by recording eye movements in the scanner and confirming that participants maintained fixation on the cue during this period (see Materials and Methods).

Brain areas selectively responsive to the spatial and emotional cue information were identified via the main effects of the spatial cue information (informative and uninformative) by emotional cue information (negative, uninformative, and neutral) ANOVA. The main effect of spatial information, depicted in Figure $2 A$ and Table 2, produced activations in the canonical spatial attention network, including the superior parietal lobule (SPL), IPS/inferior parietal lobule (IPL), and the FEF $(p<0.05$, corrected). All these regions showed greater activation for spatially informative than spatially uninformative cues. This activation pattern is similar to that observed in previous studies of spatial attention (Gitelman et al., 1999; Mesulam, 1999; Corbetta and Shulman, 2002; Egner et al., 2008). In addition, spatial cuing effects were observed in ventral visual regions, particularly the fusiform gyrus (FG), which is involved in face perception (Kanwisher et al., 1997) and has been shown to be modulated by covert attention toward face stimuli (Wojciulik et al., 1998).

The main effect of emotional cue information evoked activations in a set of limbic, cortical, and subcortical regions including the posterior cingulate cortex, orbitofrontal cortex, subcallosal gyrus of the anterior cingulate cortex (ACC), ACC, and IPS/IPL (Fig. $2 C$, Table 2) ( $p<0.05$, corrected). The pattern of cuerelated activity in these regions was negative $>$ uninformative $>$ neutral. Emotional cue information-related effects were also observed in the insula and the fusiform gyrus (Fig. 2C, Table 2) $(p<$ 0.05 , corrected). In these regions, the pattern of cue-related activity was negative $>$ neutral $>$ uninformative. In addition, region of interest (ROI) analyses revealed negative $>$ neutral $>$ uninformative emotional cue-related activity in the amygdala (Fig. 2C) (coordinates: $x=18, y=2, z=-16$ ).

Finally, we examined regions that showed an interaction of spatial and emotion cue-related information. Such an interaction was observed in bilateral parahippocampal gyrus (Table 2) $(p<$ 0.05 , corrected). Simple effects tests conducted to examine the pattern of interaction in bilateral parahippocampal gyrus showed that that there was increased activity for negative relative to neutral and uninformative cues when the cues were spatially informative $(p<0.05)$ but not when they were spatially uninformative $(p>0.1)$. In addition, ROI analyses showed an interaction of spatial and emotional cue information in the PCC (Fig. $2 B$ ) (coordinates: $x=-5, y=-45, z=44$ ) such that the PCC was activated by spatially informative cues only when the cue also signaled angry faces (supplemental Fig. 1, available at www.jneurosci.org as supplemental material). Thus, it seems that the PCC was involved in spatial orienting only when the attentional target was high in emotional or motivational relevance.

In summary, our results implicate the spatial attention network, including the SPL, IPS/IPL, and FEF in the spatial biasing of attention. The cingulate component appears to contribute to the spatial attention network only when the target has high emotional or motivational relevance. Finally, emotion information-related biasing of attention implicated limbic and subcortical regions including posterior cingulate cortex, amygdala, orbitofrontal cortex, subcallosal gyrus, and ACC, as well as cortical regions such as insular cortex, fusiform gyrus, and IPS/IPL.

\section{Table 2. Peak activations for cue-related effects}

\begin{tabular}{|c|c|c|c|}
\hline Regions & $\mathrm{BA}$ & Coordinates & Zscore \\
\hline \multicolumn{4}{|l|}{ Spatial cue information } \\
\hline \multirow[t]{2}{*}{ Frontal eye field } & 6 & $-24,-8,56$ & 7.42 \\
\hline & 6 & $30,-4,56$ & 4.82 \\
\hline Middle frontal gyrus & 6 & $-4,8,54$ & 5.89 \\
\hline Precentral gyrus & 6 & $-48,-2,40$ & 4.98 \\
\hline \multirow[t]{2}{*}{ Superior parietal lobule } & 7 & $22,-62,66$ & 5.56 \\
\hline & 7 & $-16,-64,58$ & 4.35 \\
\hline \multirow[t]{2}{*}{ Intraparietal sulcus/inferior parietal lobule } & 40 & $-46,-40,54$ & 3.72 \\
\hline & 40 & $44,-32,44$ & 3.49 \\
\hline \multirow[t]{2}{*}{ Fusiform gyrus } & 19 & $-42,-72,-10$ & 6.14 \\
\hline & 37 & $44,-60,-8$ & 5.82 \\
\hline \multicolumn{4}{|l|}{ Emotional cue information } \\
\hline Anterior cingulate gyrus & 24 & $16,-14,38$ & 4.23 \\
\hline Subcallosal gyrus & 32 & $2,24,-18$ & 3.80 \\
\hline Orbitofrontal cortex & 10 & $-14,38,-18$ & 4.22 \\
\hline Posterior cingulate cortex & 31 & $-10,-44,38$ & 3.94 \\
\hline Inferior temporal gyrus & 20 & $54,-26,-18$ & 3.71 \\
\hline Insula & 13 & $32,14,-18$ & 3.60 \\
\hline \multirow[t]{2}{*}{ Fusiform gyrus } & 19 & $-40,-68,-10$ & 3.87 \\
\hline & 19 & $-32,-88,-10$ & 4.60 \\
\hline \multirow[t]{2}{*}{ Inferior parietal lobule/intraparietal sulcus } & 40 & $-38,-44,46$ & 3.22 \\
\hline & & $-32,-48,50$ & 3.07 \\
\hline \multicolumn{4}{|l|}{$\begin{array}{l}\text { Interaction of spatial and emotional cue } \\
\text { information }\end{array}$} \\
\hline \multirow[t]{2}{*}{ Parahippocampal gyrus } & 27 & $10,-32,-2$ & 3.97 \\
\hline & 30 & $-12,-40,2$ & 3.89 \\
\hline \multicolumn{4}{|l|}{$\begin{array}{l}\text { Conjunction of spatial and emotional cue } \\
\text { information }\end{array}$} \\
\hline Frontal eye field & 6 & $-22,-12,58$ & 2.93 \\
\hline \multirow[t]{3}{*}{ Inferior parietal lobule/intraparietal sulcus } & 40 & $-32,-48,50$ & 3.07 \\
\hline & & $-40,-38,48$ & 2.71 \\
\hline & & $42,-32,38$ & 2.98 \\
\hline \multirow[t]{2}{*}{ Superior parietal lobule } & 7 & $-14,-62,56$ & 2.97 \\
\hline & & $-22,-68,56$ & 2.62 \\
\hline \multirow[t]{4}{*}{ Fusiform gyrus } & 19 & $-44,-80,-12$ & 2.79 \\
\hline & & $42,-78,-6$ & 2.65 \\
\hline & & $36,-70,-12$ & 2.69 \\
\hline & & $-38,-68,-14$ & 3.86 \\
\hline Precentral gyrus & 4 & $-40,-20,52$ & 3.00 \\
\hline Middle occipital gyrus & 18 & $-28,-82,6$ & 2.95 \\
\hline
\end{tabular}

Integration of spatial and emotional cue-related effects

Next, we used conjunction analyses (Friston et al., 2005; Nichols et al., 2005) to examine regions that jointly use spatial and emotional cue-related information to guide spatial attention. These analyses revealed joint effects of spatial and emotional cuerelated information in the spatial attention network including SPL, IPS/IPL, and FEF as well as in the fusiform gyrus (Table 2). The mean parameter estimates for each cue condition for these ROIs are depicted in Figure $3 B$ to further describe how spatial and emotion-related information is represented in these regions. Cue-related BOLD responses in SPL, IPS/IPL, and FEF (Fig. 3B; supplemental Fig. 1, available at www.jneurosci.org as supplemental material) displayed an inverse pattern to that depicted in the behavioral data. SPL, IPS/IPL, and FEF showed greater activation for spatially informative than uninformative cues as well as negative $>$ uninformative $>$ neutral emotional cue-related activation. Although it appears that the effect of emotional-cue related information was most pronounced in the presence of spatial information (Fig. 3B), this interaction was not significant for any of the regions showing a conjunction of spatial and emotional cue-related effects (all interaction values of $p>0.1$ ) indicating that spatial and emotional cue-related effects in SPL, IPS/ 
A

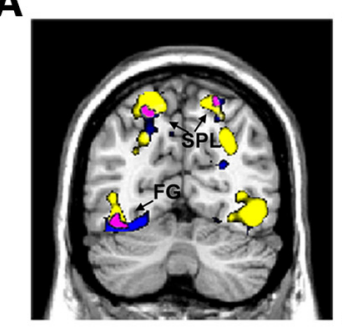

B
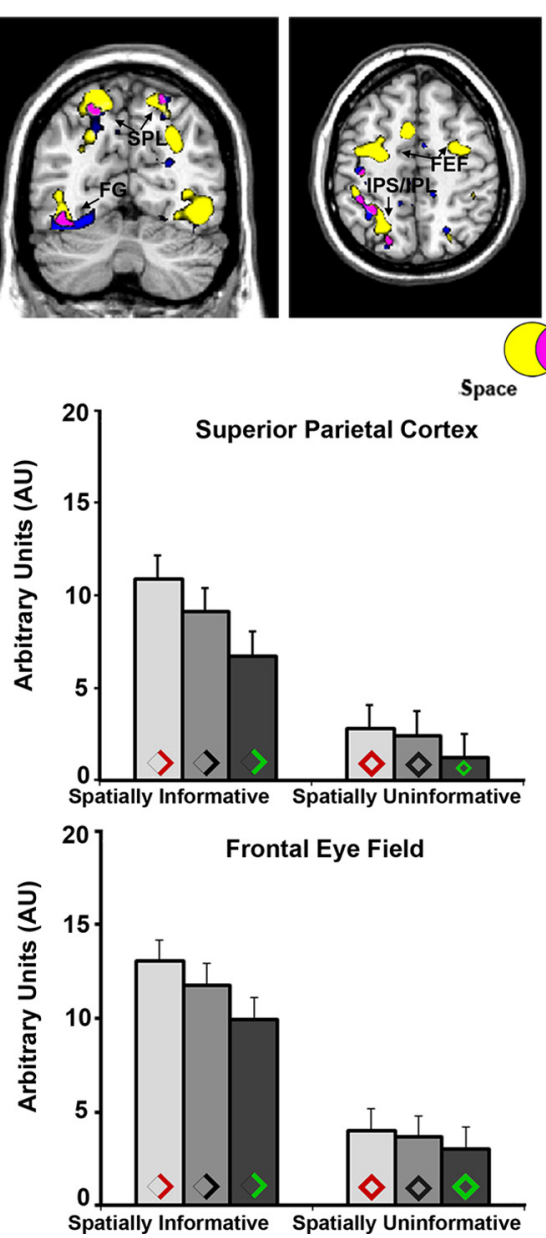
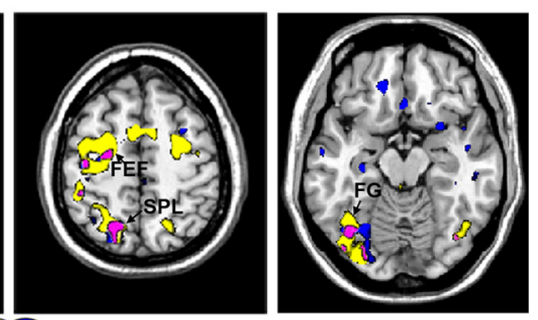

Emotion

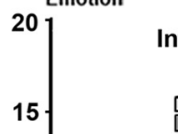

Inferior Parietal Cortex

Emotionally Negative Cue Emotionally Uniformative Cue Emotionally Neutral Cue
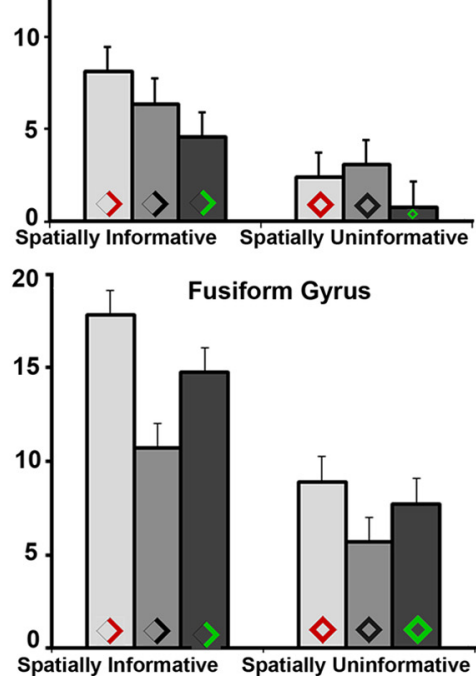

Figure 3. $A$, Images from the conjunction analysis depict brain areas (in pink) that are responsive to both spatial and emotional cue information. Regions include SPL, FG, IPS/IPL, and FEF. $\boldsymbol{B}$, Mean $\beta$ parameter estimates are depicted as a function of spatial and emotional cue information in regions showing a conjunction of the two effects. SPL, IPS/IPL, FEF, and FG show additive effects of spatial and emotional cue information indicating that they play an important role in integrating spatial and emotional saliencybased representations. Error bars represent within-subject SEM.

IPL, and FEF were independent. Finally, although the fusiform gyrus also showed a conjunction of spatial and emotional expectancies, it showed a different pattern of results than frontal and parietal regions with greater BOLD response for negative and neutral cues and lesser activity for the uninformative emotional cue.

In summary, our results demonstrate that regions comprising the spatial attention network, including IPS/IPL, FEF, and SPL, show additive effects of spatial and emotional cue information, indicating that they play an important role in integrating spatial and emotional saliency-based representations. In addition, our results showed an overlap of spatial and emotional expectancies in the fusiform gyrus, which is an important target of top-down biasing of attention by frontoparietal regions, specifically for the face-related task at hand (Egner and Hirsch, 2005).

\section{Emotion-related modulation of spatial biasing of attention}

Our next aim was to investigate whether the spatial attention network mediates anticipatory shifts in spatial attention differentially for emotional and nonemotional cues. To do this, we computed the correlation between neural activity for spatially valid cues and behavioral cue benefits derived from valid spatial cues on a trial-by-trial basis, which resulted in parameter estimates (for details, see Materials and Methods). We then averaged the parameter estimates separately for emotionally negative, unin- formative, and neutral cues, and examined their pattern in regions that comprised the spatial attention network. In Figure 4, the pattern of mean parameter estimates across subjects (for pattern of correlation for a representative subject, see supplemental Fig. 2, available at www.jneurosci.org as supplemental material) indicates a stronger positive correlation between brain activity and spatial cue benefits derived from negative cues, no correlation for uninformative emotional cues, and a negative correlation between brain activity and spatial cue benefits derived from neutral cues in the SPL $\left(F_{(2,11)}=6.23 ; p<0.01\right)$, IPS/IPL $\left(F_{(2,11)}=8.27 ; p<0.01\right)$, and fusiform gyrus $\left(F_{(2,11)}=4.22 ; p<0.05\right)$. In summary, activation in fusiform gyrus and components of the spatial attention network such as SPL and IPS/IPL was more positively correlated with the speed of attentional shifts to angry faces, whereas it was negatively correlated with speed of attentional shifts to neutral faces. These results indicate that the spatial attention network enhances the impact of motivationally relevant information and diminishes the impact of motivationally less relevant information.

Brain connectivity during emotional cuing After establishing the role of the spatial attention network in spatial biasing of attention and limbic regions, including amygdala and PCC in emotion-related biasing of attention, we examined the possibility that limbic input to the spatial attention network and inferotemporal visual areas facilitates visual search for motivationally significant events. For this purpose, we used PPI analyses (see Materials and Methods), which allowed us to examine context-specific changes in functional integration between amygdala and PCC and regions involved in emotional and spatial biasing of attention. PPI analysis was conducted using functional amygdala and PCC ROIs that showed a main effect of emotional cuing. Figure 5 shows that amygdala activity during preparation for visual search for angry targets (angry cues $>$ uninformative cues) was associated with a simultaneous trial-by-trial increase in activity in the FEF (peak MNI coordinate: $-26,-10,46$ ), IPL $(48,-32,48 ;-48,-34,46)$, and the fusiform gyrus $(-40,-58$, $-18 ; 32,-60,-12)$. No task-related changes in connectivity were noted for the PCC ROI. In summary, our results show that the functional coupling between amygdala and regions involved in spatial biasing of attention (FEF and IPL) and in face processing (fusiform gyrus) increased for negative cues that indicated upcoming angry targets compared with emotionally uninformative cues that provided no information regarding the upcoming targets, supporting the possibility that enhanced amygdala input to the spatial attention network and inferotemporal visual areas facilitates rapid detection of upcoming motivationally significant events. 


\section{Discussion}

In the present study, we investigated how the brain uses expectations regarding the motivational significance of upcoming environmental events to guide spatial attention. In contrast to previous studies that have examined the exogenous capture of attention by the emotional/ motivational salience of visual stimuli, the present study implemented a novel task that manipulated the endogenous guidance of attention by predictive cues that offered probabilistic information related to the location and emotional salience of an upcoming stimulus. As expected, spatially valid cues enhanced the detection of targets. A novel finding was that cues validly predicting angry faces as the target resulted in faster detection times than uninformative cues followed by angry faces, indicating that the acceleration of spatial attention by a cue predicting an emotionally salient event can be endogenously mediated and is not solely dependent on bottom-up target features.

How are behavioral cue benefits for motivationally significant targets achieved? Analysis of cue-related BOLD responses implicated the spatial attention network, including the SPL, IPS/IPL, and FEF in the spatial biasing of attention, whereas emotional biasing of attention also implicated limbic and subcortical regions including the PCC, amygdala, orbitofrontal cortex, subcallosal gyrus, and ACC. Furthermore, the posterior cingulate component of the spatial attention network was activated predominantly by spatially informative cues when the cue also signaled angry face targets. Importantly, we found that the fusiform gyrus and spatial attention network, including IPS/ IPL, FEF, and SPL, display additive effects of spatial and emotional cue information, indicating that they play an important role in integrating spatial and emotional saliency-based representations. Finally, these frontoparietal spatial attention network nodes also showed increased connectivity with the amygdala after angry face cues, suggesting that the expectation of a threatening stimulus enhances amygdala input to the spatial attention network and inferotemporal visual areas, presumably in the service of facilitating the rapid detection of upcoming motivationally significant events.

It is not surprising that our results showed robust activation in the frontoparietal components of the spatial attention network in response to spatially informative compared with uninformative cues. Lack of activation in the posterior cingulate component for spatial cuing was unexpected considering that the PCC has been hypothesized to play an important role in the generation of visual spatial biases and is considered a major component of the spatial attention network (Mesulam, 1981; Hopfinger et al., 2000; Small et al., 2003). However, additional analysis revealed that the PCC was activated selectively by spatial cues that also signaled angry faces. Thus, it seems that PCC involvement in anticipatory biasing of spatial attention is more specialized for targets with high emotional relevance. This finding is in line with single-cell studies showing that PCC neurons signal expected and actual reward outcomes associated with shifts of gaze during spa-
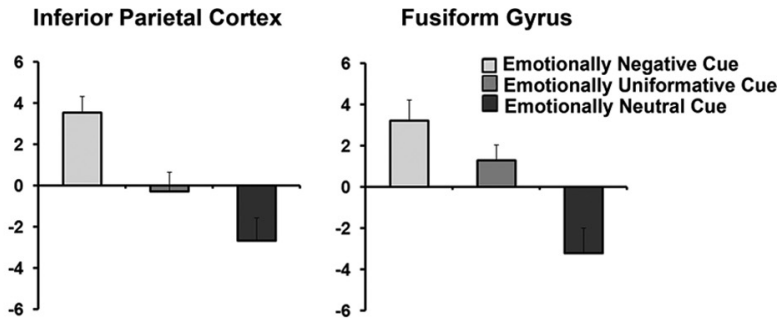

and FG and spatial cue benefits derived from emotionally negative, uninformative, and neutral cues. Mean $\beta$ parameter estimates ( \pm within-subject SEM) from SPL, IPL, and FG show a differential pattern of correlation between neural activity and the speed of attentional shifts with a stronger positive correlation
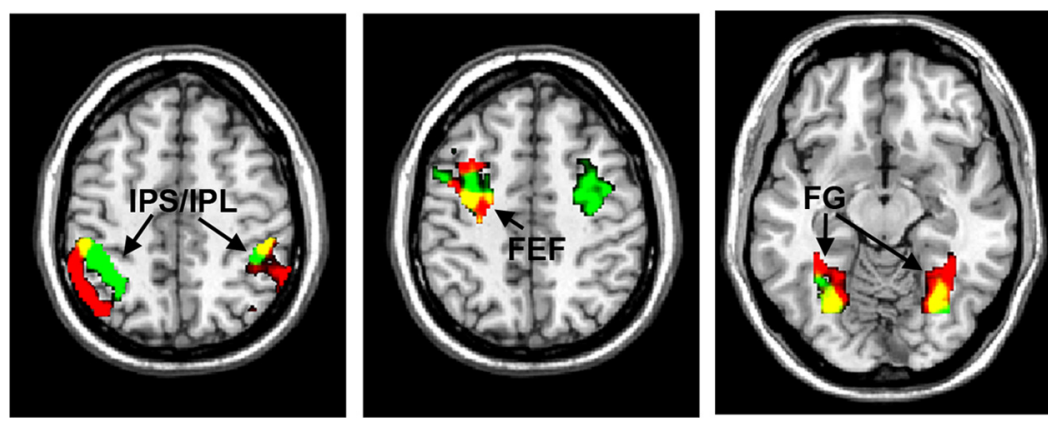

ow a task-specific increase in functional integration with the amygdala within region that show a conjunction of emotional and spatial cuing (green). The yellow areas show the overlap.

tial attention tasks (McCoy et al., 2003). Similarly, fMRI studies show PCC activity correlates positively with anticipatory shifts of spatial attention and that this relationship is strengthened by the presence of monetary incentives (Small et al., 2005) and motivationally relevant targets (Mohanty et al., 2007). Anatomically, the primate cingulate gyrus is interconnected with the amygdala, parahippocampal region, insula, OFC, and inferior parietal lobule (Mesulam et al., 1977; Pandya et al., 1981; Morecraft et al., 1992; Morris et al., 2000) providing additional support for the role of PCC as a potential conduit between the limbic system and the spatial attention network during attentional biasing toward motivationally salient information.

An important question that the present study sought to answer was how the human brain represents and integrates concurrent spatial and emotion-related information in anticipation of visual search for motivationally relevant events. Our findings highlight a key role for the frontoparietal spatial attention network in the compilation of a salience map that combines the spatial coordinates of an event with its perceptual and motivational relevance (Fecteau and Munoz, 2006; Gottlieb, 2007). This salience map provides a search template that primes locations and emotional or motivational features that are consistent with the endogenously provided information. For example, spatial expectancies could prime IPS neurons whose receptive fields overlap with the anticipated target location (Moran and Desimone, 1985; Treue and Maunsell, 1996), whereas emotional expectancies could prime IPS neurons responsive to the emotionally or motivationally relevant visual features (Bermpohl et al., 2006), resulting in an additive priming effect in neurons responsive to the target feature that also have receptive fields overlapping the attended target location (Treue and Martínez Trujillo, 1999). This role of the frontoparietal spatial attention network in compiling a salience map is consistent with previous studies showing its involvement in processing the motiva- 
tional relevance of extrapersonal stimuli (Mountcastle et al., 1975; Gottlieb et al., 1998; Platt and Glimcher, 1999; Sugrue et al., 2004; Mohanty et al., 2008) and integrating spatial and feature-based sources of information to guide attention (Egner et al., 2008).

Our results also revealed an overlap of spatial and emotional expectancies in the fusiform gyrus, which is an important target of top-down biasing of attention by frontoparietal regions, specifically for the face-related task at hand (Egner and Hirsch, 2005). Additionally, the neural activation in fusiform gyrus and parietal components of the spatial attention network correlated positively with the speed of attentional shifts to angry faces and negatively with attentional shifts to neutral faces. This pattern indicates that the spatial attention network modulates attention in a way that enhances the impact of motivationally relevant stimuli and diminishes the impact of motivationally less relevant stimuli (Mohanty et al., 2008). Although present findings apply to cues that are indicative of threatening targets, these results are similar to results obtained for motivationally salient stimuli that are rewarding in nature (Small et al., 2003; Mohanty et al., 2008).

Another question addressed in the present study was whence the spatial attention network receives information about the emotional and motivational relevance of attentional targets. Our results show that limbic regions including PCC, amygdala, orbitofrontal cortex, and subcallosal gyrus show increased activity for cues predicting threatening targets compared with neutral and uninformative cues. Furthermore, among these regions, the amygdala shows greater functional coupling with regions involved in spatial biasing of attention (FEF and IPL) and in face processing (fusiform gyrus) for cues indicating upcoming angry targets compared with cues that provide no information regarding the emotional content of upcoming targets. Hence, it is likely that expectation of a threatening stimulus is encoded by limbic regions, particularly the amygdala, which in turn modulates activity in the spatial attention network and inferotemporal visual areas to facilitate the rapid detection of the relevant event.

This proposed role for the amygdala in biasing the spatial attention network and inferotemporal visual areas is consistent with studies demonstrating its role in assessing the motivational salience of attentional targets (Pessoa et al., 2002; Vuilleumier et al., 2004; Vuilleumier and Pourtois, 2007). Anatomical tracing studies show dense feedback connections from the amygdala to the cortical sensory areas (Amaral and Price, 1984; Amaral et al., 2003). In humans, enhanced neural response in fusiform face area (FFA) for angry compared with neutral faces (Vuilleumier et al., 2004; Sabatinelli et al., 2005) is highly correlated with amygdala activity (Morris et al., 1998; Pessoa et al., 2002; Sabatinelli et al., 2005) and abolished in the individuals with amygdala lesions (Vuilleumier et al., 2004), providing additional support for the role of the amygdala in attention-related modulation of inferotemporal cortices.

Although direct anatomical connections from the amygdala to frontoparietal components of the spatial attention network are sparse at best, the amygdala shows extensive anatomical connectivity with the cingulate component of the spatial attention network, raising the possibility that the cingulate gyrus acts as the principal relay for conveying information on motivational salience to the spatial attention network (Mesulam et al., 1977). In the present study, we saw frontoparietal involvement for spatial biasing of attention, with PCC involvement occurring only when the cues were motivationally salient. Both amygdala and PCC showed increased activity during emotional biasing of attention, but only amygdala showed greater functional coupling with frontoparietal spatial attention network and inferotemporal visual areas for cues indicating angry targets. It is possible that the PCC is as tightly interconnected with the amygdala during attentional shifts to motivationally salient as to neutral events so that there would be no change in effective connectivity to detect. However, specific amygdala signals announcing an emotionally significant upcoming event could increase the shunting of this information to other components of the spatial attention network. This hypothetical arrangement would be consistent with our data, namely an enhancement of effective connectivity from the amygdala to the FEF and IPS but not the PCC in response to cues predicting threatening faces.

In conclusion, our study implements a novel experimental paradigm to demonstrate that expectancies regarding probable locations and motivational relevance of upcoming search targets aid faster detection of these targets. Preparatory cue-related neural responses demonstrate how spatial and emotional saliencerelated information are integrated in key regions of the spatial attention network, suggesting a role for these regions in the generation of a "top-down salience map" that guides visual attention. Finally, augmented connectivity of these frontoparietal regions and FFA with the amygdala after cues predicting angry face targets suggest that active search for a threatening stimulus elicits amygdala input to the spatial attention network and inferotemporal visual areas, facilitating the rapid detection of upcoming motivationally significant events.

\section{References}

Amaral DG, Price JL (1984) Amygdalo-cortical projections in the monkey (Macaca fascicularis). J Comp Neurol 230:465-496.

Amaral DG, Behniea H, Kelly JL (2003) Topographic organization of projections from the amygdala to the visual cortex in the macaque monkey. Neuroscience 118:1099-1120.

Armony JL, Dolan RJ (2002) Modulation of spatial attention by fearconditioned stimuli: an event-related fMRI study. Neuropsychologia 40:817-826.

Bermpohl F, Pascual-Leone A, Amedi A, Merabet LB, Fregni F, Gaab N, Alsop D, Schlaug G, Northoff G (2006) Dissociable networks for the expectancy and perception of emotional stimuli in the human brain. Neuroimage 30:588-600.

Corbetta M, Shulman GL (2002) Control of goal-directed and stimulusdriven attention in the brain. Nat Rev Neurosci 3:201-215.

Corbetta M, Kincade JM, Ollinger JM, McAvoy MP, Shulman GL (2000) Voluntary orienting is dissociated from target detection in human posterior parietal cortex. Nat Neurosci 3:292-297.

Dale AM (1999) Optimal experimental design for event-related fMRI. Hum Brain Mapp 8:109-114.

Eastwood JD, Smilek D, Merikle PM (2001) Differential attentional guidance by unattended faces expressing positive and negative emotion. Percept Psychophys 63:1004-1013.

Egner T, Hirsch J (2005) Cognitive control mechanisms resolve conflict through cortical amplification of task-relevant information. Nat Neurosci 8:1784-1790

Egner T, Monti JM, Trittschuh EH, Wieneke CA, Hirsch J, Mesulam MM (2008) Neural integration of top-down spatial and feature-based information in visual search. J Neurosci 28:6141-6151.

Fecteau JH, Munoz DP (2006) Salience, relevance, and firing: a priority map for target selection. Trends Cogn Sci 10:382-390.

Friston KJ, Buechel C, Fink GR, Morris J, Rolls E, Dolan RJ (1997) Psychophysiological and modulatory interactions in neuroimaging. Neuroimage 6:218-229.

Friston KJ, Penny WD, Glaser DE (2005) Conjunction revisited. Neuroimage 25:661-667.

Gitelman DR, Nobre AC, Parrish TB, LaBar KS, Kim YH, Meyer JR, Mesulam M (1999) A large-scale distributed network for covert spatial attention: further anatomical delineation based on stringent behavioural and cognitive controls. Brain 122:1093-1106. 
Gitelman DR, Penny WD, Ashburner J, Friston KJ (2003) Modeling regional and psychophysiologic interactions in fMRI: the importance of hemodynamic deconvolution. Neuroimage 19:200-207.

Gottlieb J (2007) From thought to action: the parietal cortex as a bridge between perception, action, and cognition. Neuron 53:9-16.

Gottlieb JP, Kusunoki M, Goldberg ME (1998) The representation of visual salience in monkey parietal cortex. Nature 391:481-484.

Hahn S, Gronlund SD (2007) Top-down guidance in visual search for facial expressions. Psychon Bull Rev 14:159-165.

Hopfinger JB, Buonocore MH, Mangun GR (2000) The neural mechanisms of top-down attentional control. Nat Neurosci 3:284-291.

Kanwisher N, McDermott J, Chun MM (1997) The fusiform face area: a module in human extrastriate cortex specialized for face perception. J Neurosci 17:4302-4311.

Kastner S, Pinsk MA, De Weerd P, Desimone R, Ungerleider LG (1999) Increased activity in human visual cortex during directed attention in the absence of visual stimulation. Neuron 22:751-761.

Lamy D, Amunts L, Bar-Haim Y (2008) Emotional priming of pop-out in visual search. Emotion 8:151-161.

McCoy AN, Crowley JC, Haghighian G, Dean HL, Platt ML (2003) Saccade reward signals in posterior cingulate cortex. Neuron 40:1031-1040.

Mesulam MM (1981) A cortical network for directed attention and unilateral neglect. Ann Neurol 10:309-325.

Mesulam MM (1999) Spatial attention and neglect: parietal, frontal and cingulate contributions to the mental representation and attentional targeting of salient extrapersonal events. Philos Trans R Soc Lond B Biol Sci 354:1325-1346.

Mesulam MM, Van Hoesen GW, Pandya DN, Geschwind N (1977) Limbic and sensory connections of the inferior parietal lobule (area PG) in the rhesus monkey: a study with a new method for horseradish peroxidase histochemistry. Brain Res 136:393-414.

Mogg K, Bradley BP (1999) Orienting of attention to threatening facial expressions presented under conditions of restricted awareness. Cogn Emot 13:713-740.

Mohanty A, Herrington JD, Koven NS, Fisher JE, Wenzel EA, Webb AG, Heller W, Banich MT, Miller GA (2005) Neural mechanisms of affective interference in schizotypy. J Abnorm Psychol 114:16-27.

Mohanty A, Engels AS, Herrington JD, Heller W, Ho MH, Banich MT, Webb AG, Warren SL, Miller GA (2007) Differential engagement of anterior cingulate cortex subdivisions for cognitive and emotional function. Psychophysiology 44:343-351.

Mohanty A, Gitelman DR, Small DM, Mesulam MM (2008) The spatial attention network interacts with limbic and monoaminergic systems to modulate motivation-induced attention shifts. Cereb Cortex 18: $2604-2613$

Moran J, Desimone R (1985) Selective attention gates visual processing in the extrastriate cortex. Science 229:782-784.

Morecraft RJ, Geula C, Mesulam MM (1992) Cytoarchitecture and neural afferents of orbitofrontal cortex in the brain of the monkey. J Comp Neurol 323:341-358.

Morris JS, Ohman A, Dolan RJ (1998) Conscious and unconscious emotional learning in the human amygdala. Nature 393:467-470.
Morris R, Paxinos G, Petrides M (2000) Architectonic analysis of the human retrosplenial cortex. J Comp Neurol 421:14-28.

Mountcastle VB, Lynch JC, Georgopoulos A, Sakata H, Acuna C (1975) Posterior parietal association cortex of the monkey: command functions for operations within extrapersonal space. J Neurophysiol 38:871-908.

Nichols T, Brett M, Andersson J, Wager T, Poline JB (2005) Valid conjunction inference with the minimum statistic. Neuroimage 25:653-660.

Ollinger JM, Shulman GL, Corbetta M (2001a) Separating processes within a trial in event-related functional MRI. Neuroimage 13:210-217.

Ollinger JM, Corbetta M, Shulman GL (2001b) Separating processes within a trial in event-related functional MRI. Neuroimage 13:218-229.

Pandya DN, Van Hoesen GW, Mesulam MM (1981) Efferent connections of the cingulate gyrus in the rhesus monkey. Exp Brain Res 42:319-330.

Pessoa L, McKenna M, Gutierrez E, Ungerleider LG (2002) Neural processing of emotional faces requires attention. Proc Natl Acad Sci U S A 99:11458-11463.

Platt ML, Glimcher PW (1999) Neural correlates of decision variables in parietal cortex. Nature 400:233-238.

Pourtois G, Grandjean D, Sander D, Vuilleumier P (2004) Electrophysiological correlates of rapid spatial orienting towards fearful faces. Cereb Cortex 14:619-633.

Sabatinelli D, Bradley MM, Fitzsimmons JR, Lang PJ (2005) Parallel amygdala and inferotemporal activation reflect emotional intensity and fear relevance. Neuroimage 24:1265-1270.

Small DM, Gitelman DR, Gregory MD, Nobre AC, Parrish TB, Mesulam MM (2003) The posterior cingulate and medial prefrontal cortex mediate the anticipatory allocation of spatial attention. Neuroimage 18:633-641.

Small DM, Gitelman D, Simmons K, Bloise SM, Parrish T, Mesulam MM (2005) Monetary incentives enhance processing in brain regions mediating top-down control of attention. Cereb Cortex 15:1855-1865.

Sugrue LP, Corrado GS, Newsome WT (2004) Matching behavior and the representation of value in the parietal cortex. Science 304:1782-1787.

Tipples J, Atkinson AP, Young AW (2002) The eyebrow frown: a salient social signal. Emotion 2:288-296.

Treue S, Martínez Trujillo JC (1999) Feature-based attention influences motion processing gain in macaque visual cortex. Nature 399:575-579.

Treue S, Maunsell JH (1996) Attentional modulation of visual motion processing in cortical areas MT and MST. Nature 382:539-541.

Vuilleumier P, Pourtois G (2007) Distributed and interactive brain mechanisms during emotion face perception: evidence from functional neuroimaging. Neuropsychologia 45:174-194.

Vuilleumier P, Richardson MP, Armony JL, Driver J, Dolan RJ (2004) Distant influences of amygdala lesion on visual cortical activation during emotional face processing. Nat Neurosci 7:1271-1278.

Wager TD, Nichols TE (2003) Optimization of experimental design in fMRI: a general framework using a genetic algorithm. Neuroimage 18:293-309.

Wojciulik E, Kanwisher N, Driver J (1998) Covert visual attention modulates face-specific activity in the human fusiform gyrus: fMRI study. J Neurophysiol 79:1574-1578. 